SCIREA Journal of Clinical Medicine

ISSN: 2706-8870

http://www.scirea.org/journal/CM

February 9, 2022

Volume 7, Issue 1, February 2022

https://doi.org/10.54647/cm32760

\title{
Clinical results in treatment of patients with spinal cord injuries, by transplantation of olfactory mucosa autografts: a case-series
}

Christos A. Gogos', Lucas M. Moschos², Agis D. Komis², George V. Bagias³, Dimitrios C. Papacostantinou $^{3}$, Anestis A.Charalampopoulos ${ }^{3}$

${ }^{1}$ Attikon University Hospital, Athens, GR

${ }^{2}$ Mediterraneo Hospital, Athens, GR

${ }^{3}$ National and Kapodistrian University of Athens, GR

Corresponding author: Anestis Charalampopouos, As. Prof. of Surgery, $3^{\text {nd }}$ Department of Surgery, University Hospital Attikon, Rimini Str. 1, 12462 Athens, Greece

Tel.: +302105831000

Email: achalaral@med.uoa.gr

\begin{abstract}
Introduction: Chronic traumatic Spinal Cord Injuries (SCI), leave a significant percentage of permanent motor and sensory disability. Transplantation of Olfactory Mucosa Autografts (OMA) at the lesion site is a hopeful procedure. We present a case-series of patients with SCI treated with OMA.

Material and methods: The AIS scale was utilized for neurologic evaluation. The features of the spinal cord lesion were determined by MRI. Neurophysiological studies were used in patients with clinical improvement. A program of rehabilitation after surgery was used at least for one year.
\end{abstract}


Results: Twenty patients underwent OMA (17 males, 3 females, age range: 22-40 years, mean age: $29.25 \pm 6.6$ years). 13 patients $(13 / 20,65 \%)$ had tetraplegia and $7(7 / 20,35 \%)$ paraplegia. Overall $80 \%$ of the patients were in ASIA score A and $30 \%$ score B. During the follow-up period, 9 patients presented improvement in ASIA scale estimation $(9 / 20,45 \%) ; 8$ from $\mathrm{A}$ to $\mathrm{B}$, and 1 from $\mathrm{A}$ to $\mathrm{C}$. A complication rate of $25 \%$ was noted.All postoperative complications were minor and treated during in-hospital stay.

Conclusion: OMA in patients with SCI, offers minor clinical benefits in up to $45 \%$ of patients. Intensive rehabilitation is an important factor of maintenance and improvement of clinical and functional results obtained.

Keywords: Spinal Cord Injury, Olfactory Mucosa Autografts, Rehabilitation, complications

\section{Introduction}

According to World Health Organization report, every year worldwide, 250.000-500.000 people suffer from spinal cord injuries. The National Spinal Cord Injury Statistical Center of USA estimates that the annual incidence of spinal cord injuries is 54 cases/1.000.000 people. The majority of these patients are male (78\%), a significant percentage of which (more than $30 \%$ ) are patients with complete paraplegia or tetraplegia. In a review study, the global prevalence and incidence of traumatic spinal cord injuries, ranges from 10 to 85 cases/1.000.000 people and exhibits wide geographical and demographical variability [1].

Experimental data reveal that transplantation of olfactory mucosa following spinal cord injury promotes recovery in animal models [2]. New reviews and meta-analyses show significant functional recovery after stem cell transplantation in spinal cord injuries, while outcomes seem to be influenced by a large numbers of factors such as the number and type of transplanted cells, the site of injury, the chronicity of the injury, the type of the lesion, and the type and timing of immunosuppression [3].

The olfactory mucosa is the only part of the adult nervous system with lifelong neurogenesis and axonogenesis and can be easily accessed via minimal invasive techniques. The intact olfactory mucosal epithelium contains multipotent stem cells that play a significant role in regeneration and restoration; Olfactory ensheating cells (OECs) and neural stem cells (NSCs) 
with their important properties and regenerative capacity, are prime candidates for transplantation, in patients with spinal cord injuries [4].

Olfactory mucosa autografts in human spinal cord injuries is a new technique first documented in 2006 by Carlos Lima et al. [5]. In this study, a benefit was demonstrable in what regards the scores of the ASIA motor scale, bladder filling sensation and anal sphincteric function [6].

The aim of the present study is to present the clinical results of transplantation of olfactory mucosa autografts in 20 patients with spinal cord injuries.

\section{Materials and methods}

Twenty patients with spinal cord injury, we performed transplantation of olfactory mucosa autografts (OMA). In the main neurologic evaluation of patients before and after surgery, ASIA scale was used, according to International Standards for Neurological Classification of Spinal Cord Injury (I.S.N.C.S.C.I.), using the American Spinal Injuries Association Impairment Scale (AIS). Patients were assessed by neurologic function and stratified according to the AIS Impairment scale in group A or group B. The length of lesion was registered in MRI.

The following eligibility criteria for transplantation of olfactory mucosa were used; complete sensory or motor dysfunction (ASIA A or B). The length of lesion by MRI, was $<3 \mathrm{~cm}$ in cervical spinal cord injuries, $<4 \mathrm{~cm}$ for lesions T1-T10 and no lesions higher than $\mathrm{C} 4$ level. Exclusion criteria were the presence of severe neurologic deficits, severe ear-nose-throat diseases, the presence of severe systemic disease and pregnancy.

All surgical procedures are performed with the same team of neurosurgeons and ENTsurgeons. The patient under general anesthesia is placed in modified park bench position. Surgical bed allows the lateral decubitus position of the patient and replacement during surgery. The head is stabilized with Mayfield support. A lumbo-spinal catheter is placed and remains $48 \mathrm{~h}$ postoperatively. The surgical operation is performed in 3 stages; in the first stage, the neurosurgeon exposes the wounded spinal cord with a midline incision, followed by laminectomy and opening of the hard twins. The wounded spinal cord is approached by posterior myelotomy in midline, where is necessary and feasible is performed posterior-lateral detection of the spinal cord. Scar tissue is removed without injuring the remnant healthy 
spinal cord. The second stage was performed by ENT-surgeon. Olfactory mucosal transplant is retrieved via transnasal endoscopic access. Vasoconstrictive drugs (adrenaline1\%) are injected in mucosa after clearing of nasal and olfactory space. A sub-mucosal and peri-ostic channel was created at the most posterior and superior segment of the internal diaphragmatic surface of the olfactory sinus and a significant amount of tissue is taken, in order to fill the cavity of the spinal cord and allow the histological and micribiological examination of the specimen. The third stage is the transplantation phase. The transplant is impregnated in cerebrospinal fluid and is sliced in small pieces in order to increase the surface of the transplanted tissue. The placement of the cells starts and ends with the complete removal of the scarred area which is then tightly closed with 8-0 silk. After ensuring adequate hemostasis, the surgical wound is closed in layers. At the end of the procedure patients are transferred to a neurological intensive care unit for 24 hours.

The clinical and laboratory follow-up of patients after surgery is performed every 6 months. Main parameters of interest are the clinical examination and ASIA scale score, neurophysiology and urodynamic studies and MRI of the spinal cord.

After the patient leaves the hospital, an intensive physiotherapy and rehabilitation program is followed [7], at least 6 hours per day for 12 months. The program is subject to adjustments, depending on the changes in mobility that may occur.

The clinical and functional benefits for patients, are obtained postoperatively, studying at times the autonomic results, medullar MRI results, motor and sensory results and the results of anal and bladder function.

\section{Results}

The majority of patients, were of male sex $(17 / 20,85 \%)$. No operation was performed before 18 months after the SCI and no lesion was older than 11yrs following the index SCI. Sixteen patients $(16 / 20,80 \%)$ in ASIA scale A and the remaining $(4 / 20,20 \%)$ in ASIA scale B. The length of lesion in MRI ranged from $1.5-4.1 \mathrm{~cm}$ with a mean length of $3.1 \mathrm{~cm}$. Fourteen with SCI had cervical lesion $(14 / 20,70 \%)$ and the remaining had thoracic lesions $(6 / 20,30 \%)$. After the operation and during the follow-up period, 9 patients presented improvement in ASIA scale estimation (9/20,45\%); 8 from A to B, and 1 from A to C. Demographic,clinical and imaging features of patients with SCI are shown in table 1. 
Table 1. Demographic, clinical and imaging features of patients with spinal cord injury (SCI), before and after olfactory mucosa autotransplantation (OMA). $\mathrm{P}=$ paraplegia, $\mathrm{T}=$ tetraplegia

\begin{tabular}{|c|c|c|c|c|c|c|c|}
\hline Patient & Sex & Age & $\begin{array}{c}\text { Years } \\
\text { after SCI }\end{array}$ & $\begin{array}{l}\text { Level of } \\
\text { SCI: } \\
\text { motor- } \\
\text { sensory }\end{array}$ & $\begin{array}{c}\text { Neurologic } \\
\text { sign; } \\
\text { P or T }\end{array}$ & $\begin{array}{l}\text { Length } \\
\text { of lesion } \\
\text { in } \mathrm{cm}\end{array}$ & $\begin{array}{l}\text { ASIA scale before- } \\
\text { after OMA }\end{array}$ \\
\hline 1 & $\mathrm{M}$ & 22 & 6 & C6-C7 & $\mathrm{T}$ & 3 & A-C \\
\hline 2 & $\mathrm{M}$ & 34 & 5 & $\mathrm{C} 3-\mathrm{C} 4$ & $\mathrm{~T}$ & 3 & A-B \\
\hline 3 & $\mathrm{M}$ & 39 & 8 & $\mathrm{~T} 2-\mathrm{T} 4$ & $\mathrm{P}$ & 4,1 & A-A \\
\hline 4 & $\mathrm{~F}$ & 24 & 2 & T6-T7 & $\mathrm{P}$ & 1,5 & A-A \\
\hline 5 & M & 32 & 4 & $\mathrm{C} 4-\mathrm{C} 5$ & $\mathrm{~T}$ & 3 & B-B \\
\hline 6 & $\mathrm{M}$ & 24 & 4 & T6-T7 & $\mathrm{P}$ & 2 & B-B \\
\hline 7 & $\mathrm{M}$ & 26 & 6 & C6-C7 & $\mathrm{P}$ & 3 & B-B \\
\hline 8 & $\mathrm{M}$ & 22 & 6 & T7-T8 & $\mathrm{P}$ & 4 & A-B \\
\hline 9 & $\mathrm{M}$ & 22 & 5 & T3-T4 & $\mathrm{P}$ & 4 & A-A \\
\hline 10 & $\mathrm{~F}$ & 30 & 8 & C6-C7 & $\mathrm{T}$ & 3 & A-B \\
\hline 11 & $\mathrm{M}$ & 24 & 5 & T6-T7 & $\mathrm{P}$ & 4 & A-B \\
\hline 12 & $\mathrm{M}$ & 22 & 4 & C6-C7 & $\mathrm{T}$ & 3 & B-B \\
\hline 13 & $\mathrm{M}$ & 40 & 10 & C5-C6 & $\mathrm{T}$ & 3 & A-A \\
\hline 14 & $\mathrm{M}$ & 40 & 11 & $\mathrm{C} 5-\mathrm{C} 7$ & $\mathrm{~T}$ & 3 & A-A \\
\hline 15 & $\mathrm{~F}$ & 32 & 9 & $\mathrm{C} 5-\mathrm{C} 6$ & $\mathrm{~T}$ & 3 & A-B \\
\hline 16 & $\mathrm{M}$ & 30 & 8 & C6-C7 & $\mathrm{T}$ & 3 & A-B \\
\hline 17 & $\mathrm{M}$ & 26 & 6 & C6-C7 & $\mathrm{T}$ & 3 & A-B \\
\hline 18 & $\mathrm{M}$ & 38 & 9 & $\mathrm{C} 5-\mathrm{C} 7$ & $\mathrm{~T}$ & 3,5 & A-A \\
\hline 19 & $\mathrm{M}$ & 35 & 10 & C4-C6 & $\mathrm{T}$ & 3,6 & A-B \\
\hline 20 & M & 23 & 4 & $\mathrm{C} 4-\mathrm{C} 5$ & $\mathrm{~T}$ & 3 & A-A \\
\hline
\end{tabular}


The results of OMA transplantation are demonstrable in the following parameters:

a) In regards to electrophysiology, new voluntary EMG responses were documented in 13 patients $(13 / 20,65 \%)$. In 4 tetraplegic and 3 paraplegic patients, voluntary muscle potentials, were retrieved in lower limp muscles. Somatosensory evoked potential testing (SSEP) was not performed preoperatively due to cost, except in one patient, which demonstrated new SSEP findings recorded at a cortical level, by palmar nerve stimulation (Figure 1).

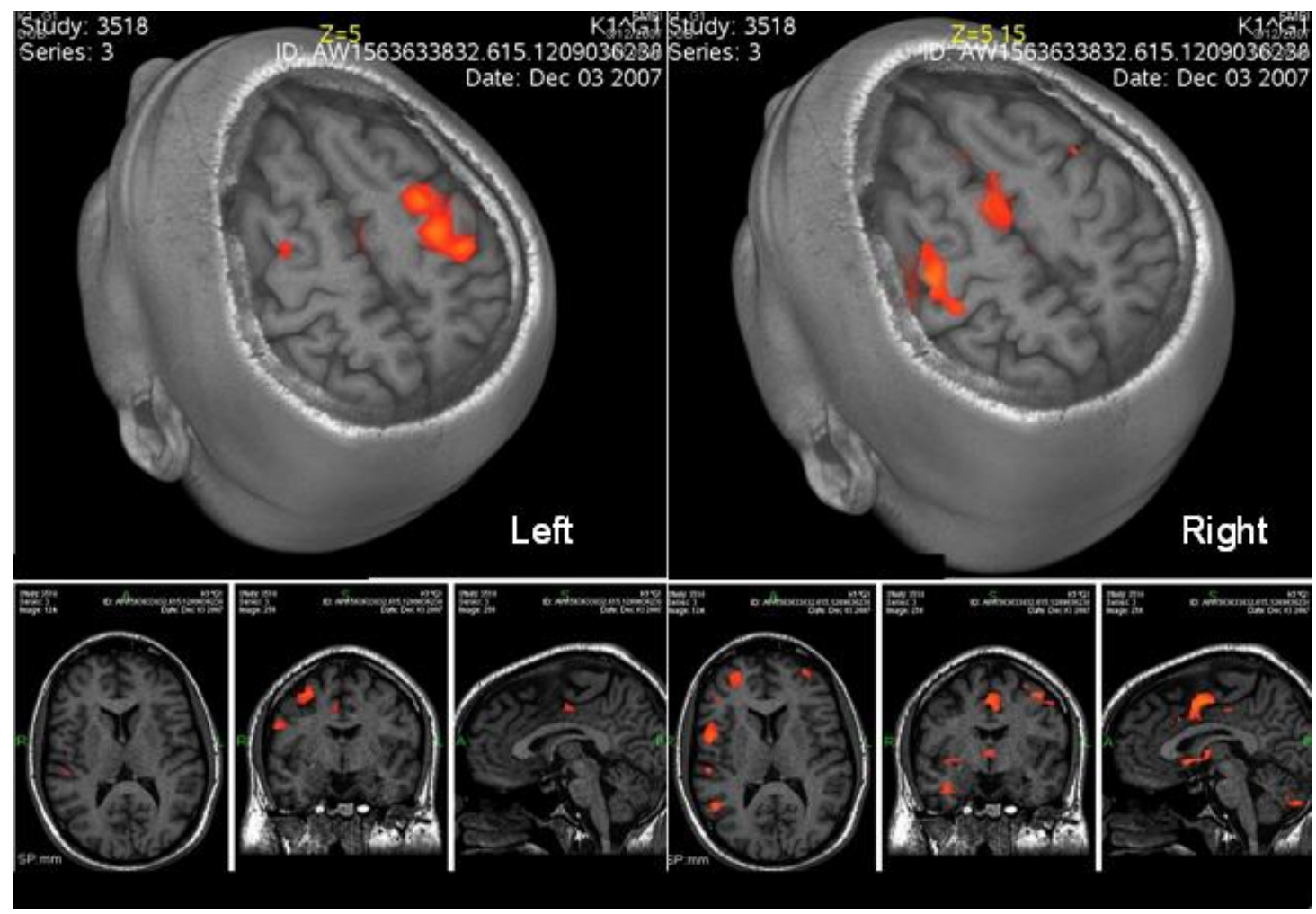

Figure1: Opening and closing of the left and right hand after palmar stimulation, f-MRI

b) Regarding anal function; Only one patient(1/20,5\%) recovered normal anal function and voluntary defecation.

c) Regarding the changes in bladder function, all patients before and after surgery required permanent bladder catheterization, although $3(15 \%)$ patients had urodynamic improvement and sensation of filling of the bladder.

Out of the 20 patients operated on, no mortality was registered, while the in-hospital stay ranged between 4-18 days. Complications were observed in 5 patients (5/20, 25\%), all of which were minor and treated during the hospital stay without long-term sequel; there was one case of subcutaneous collection of cerebrospinal fluid that resolved spontaneously, one case of pneumonia treated by antibiotics, one case of epistaxis treated with nasal packing and one case of aseptic meningitis syndrome and edema of the spinal cord. 
Concerning the biochemical characteristics of the cerebrospinal fluid, a high protein content and low glucose level was noted, with a cell count of less than 100 cells mainly of the mononuclear type. All microbiological cultures in cerebrospinal fluid and blood were sterile. A single patient manifested transient sensory loss of smell for 3 months due to nasal mucosectomy. No patient exhibited worsening of the ASIA scale score from B to A during the postoperative period.

\section{Discussion}

In this study 9 patients (45\%) demonstrated clinical benefits from OMA; in 8 patients (40\%) the underlying motor and sensory disability was improved. Indeed one tetraplegic patient with cervical lesion showed clinical improvement entailing gain of sensory and motor function in the upper and lower limbs, as well as anal sphincter function. In a report by Carlos Lima [8], a higher index of transformation from ASIA A score to ASIA C score was recorded.

The timing of treating the patients is still empiric; at least 1.5 years after the SCI and no more than 10-11 years after the SCI. In the current patient series, we didn't perform preoperative prehabilitation. This decision was implemented with the goal of reducing the overall cost of the procedure while placing emphasis on postoperative rehabilitation as a means to improve patients' clinical results.

The operation was done by the same team of neurosurgeons and ENT surgeons in all patients. Our results demonstrate that the method is safe, feasible and plausibly major adverse events. There is a consensus opinion that there is lack of sufficient neural regeneration in human SCI and therefore cellular replacements are needed to «bridge the gap», with olfactory mucosal cells being the most viable candidate [2].

The most common effective rehabilitation therapies are the BIONT (brain-initiated overground nonrobotic/nonweight supporting training) and BWSTT (Body weight-suppoted treatmill training) as they are proposed by Carlos Lima [8], the pioneer of the method of OMA for SCI. It seems that the majority of patients might benefit from preoperative and postoperative rehabilitation in regards to their functional results obtained by EMG, SSEP, MEP and urodynamic studies. Unfortunately, the improvement of functional results does not seem to correspond to clinical improvement. Moreover, it is uncertain whether the registered neurological amelioration is in fact due to the OMA procedure or the protracted course of 
postoperative rehabilitation and whether such benefits are sustainable after the cessation of postoperative physiotherapy.

In our patient sample, we consider that the f-MRI of the brain after stimulation to be an excellent diagnostic adjunct in patients showing clinical improvement. In Figure 1, there is clear brain activity, after the left and right palmar stimulation in a patient during the postoperative period. The potential benefits of such neurologic functional studies should be carefully weighed against their associated costs. In the current patient series, all patients but one, rejected postoperative f-MRI monitoring due to cost concerns.

In our patients, we didn't use preoperative rehabilitation, as all patients were young, with a satisfying general status for a surgical operation. In the postoperative period all patients followed a rehabilitation program. The R.I.C. therapy was the preferable for the following reasons: (1) Intensity: The therapy program, lasts 4-6h daily for the patient and physiotherapist, at least five days weekly. (2) Duration: There is not a well determined ending time of the program. It may lasts from one year to several years and depends from the results obtained and goals anticipated. (3) Individuality: The program of rehabilitation is strictly personal, depending from the level and severity of SCI. (4). The method RIC necessitates the active participation of the patient. The benefits of this method stem technique itself. Stretching, passive mobilization of articulations for keeping and increasing the range of motion(ROM), active exercises, electro-stimulation, massage, exercises in swimming pool, the gain of upright position and finally the re-education of walking confer substantial patientrelated benefits. (5) Psychological and neurophysiological mental functions are targeted during the program, plausibly invoking improvements in memory, speaking, attention, perception, time of reaction and time-space orientation. Postoperative rehabilitation is compulsory after OMA, as patients may often develop other secondary pathologies such as muscles shortening, stiffness of articulations, osteoporosis, decrease of muscle power and decrease of mental function.

The total number of transplanted olfactory cells may play a role in the effect size anticipated postoperatively. Cell cultivation following nasal retrieval might yield better outcomes, albeit with a significantly added cost. 


\section{Conclusion:}

OMA transplantation in patients with SCI, is a safe and feasible operation, without severe complications, in experienced and well educated surgical staff. Based on our results, up to $45 \%$ of patients are expected to benefit postoperatively, mainly in sensory functions and less in motor functions, with the postoperative rehabilitation for a long time period to be an important factor of improvement. Further studies will help answer questions regarding the length and level of lesion, the age of patients and the kind and time of rehabilitation.

\section{References}

[1] Singh A, Tetreault L, Kalsi-Ryan S, et al (2014) Global prevalence and incidence of traumatic spinal cord injury. Clin Epidemiol 6:309-331. https://doi.org/10.2147/CLEP.S68889

[2] Iwatsuki K, Yoshimine T, Kishima H, et al (2008) Transplantation of olfactory mucosa following spinal cord injury promotes recovery in rats. Neuroreport 19:1249-1252. https://doi.org/10.1097/WNR.0b013e328305b70b

[3] Abbaszadeh H-A, Niknazar S, Darabi S, et al (2018) Stem cell transplantation and functional recovery after spinal cord injury: a systematic review and meta-analysis. Anat Cell Biol 51:180-188. https://doi.org/10.5115/acb.2018.51.3.180

[4] Yao R, Murtaza M, Velasquez JT, et al (2018) Olfactory Ensheathing Cells for Spinal Cord Injury: Sniffing Out the Issues. Cell Transplant 27:879-889. https://doi.org/10.1177/0963689718779353

[5] Lima C, Pratas-Vital J, Escada P, et al (2006) Olfactory mucosa autografts in human spinal cord injury: a pilot clinical study. J Spinal Cord Med 29:191-196. https://doi.org/10.1080/10790268.2006.11753874

[6] Roberts TT, Leonard GR, Cepela DJ (2017) Classifications In Brief: American Spinal Injury Association (ASIA) Impairment Scale. Clin Orthop Relat Res 475:1499-1504. https://doi.org/10.1007/s11999-016-5133-4

[7] Cavalli L, Arcangeli M, Paladini A, et al (2019) Importance of motor rehabilitation (R.I.C) in medullary lesions in chronic phase. Surg Case Reports Rev 3:. https://doi.org/10.15761/SCRR.1000134

[8] Lima C, Escada P, Pratas-Vital J, et al (2010) Olfactory mucosal autografts and rehabilitation for chronic traumatic spinal cord injury. Neurorehabil Neural Repair 24:10-22. https://doi.org/10.1177/1545968309347685 